\title{
Transverse spectral stability of small periodic traveling waves for the KP equation
}

\author{
Mariana Haragus \\ Laboratoire de Mathématiques, Université de Franche-Comté \\ 16 route de Gray, 25030 Besançon, France \\ mharagus@univ-fcomte.fr
}

\begin{abstract}
The Kadomtsev-Petviashvili (KP) equation possesses a four-parameter family of one-dimensional periodic traveling waves. We study the spectral stability of the waves with small amplitude with respect to two-dimensional perturbations which are either periodic in the direction of propagation, with the same period as the one-dimensional traveling wave, or non-periodic (localized or bounded). We focus on the so-called KP-I equation (positive dispersion case), for which we show that these periodic waves are unstable with respect to both types of perturbations. Finally, we briefly discuss the KP-II equation, for which we show that these periodic waves are spectrally stable with respect to perturbations which are periodic in the direction of propagation, and have long wavelengths in the transverse direction.
\end{abstract}

Running head: Periodic waves of the KP equation

Keywords: KP equation, periodic traveling waves, spectral stability

\section{Contents}

1 Introduction $\quad 2$

2 One-dimensional periodic traveling waves $\quad 3$

3 The spectral stability problem $\quad 5$

$\begin{array}{lll}4 & \text { KP-I equation: periodic perturbations } & 7\end{array}$

4.1 Reformulation of the spectral stability problem . . . . . . . . . . . 7

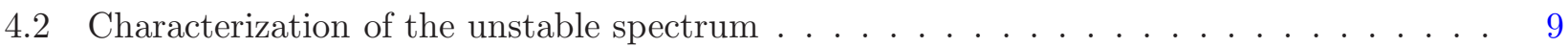

4.3 Finite and short wavelength transverse perturbations . . . . . . . . . . . . . . 10

4.4 Long wavelength transverse perturbations . . . . . . . . . . . . . . . . 10 
5.1 Bloch-wave decomposition . . . . . . . . . . . . . . . . . 15

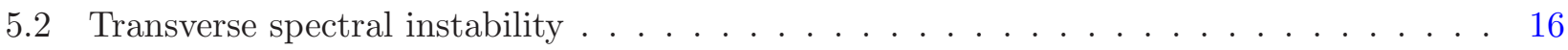

6 KP-II equation: periodic perturbations 21

$\begin{array}{llr}7 & \text { Discussion } & 23\end{array}$

References $\quad 24$

Acknowledgments This work was partially supported by the Agence Nationale de la Recherche through grant ANR PREFERED.

\section{Introduction}

We consider the Kadomtsev-Petviashvili (KP) equation

$$
\left(u_{t}-u_{x x x}-u u_{x}\right)_{x}+\sigma u_{y y}=0,
$$

in which $u(x, y, t)$ depends upon the spatial variables $x, y \in \mathbb{R}$, and the temporal variable $t \in \mathbb{R}$, and $\sigma$ is equal to either 1 or -1 . This equation is a generalization to two spatial dimensions of the well-known Korteweg-de Vries (KdV) equation

$$
u_{t}=u_{x x x}+u u_{x}
$$

and has been derived as a model in the study of the transverse stability of the line solitary waves, i.e., the stability of the solitary waves of the $\mathrm{KdV}$ equation with respect to two-dimensional perturbations [10]. In particular, it arises as a model for long waves in the water-wave problem, in the absence of surface tension when $\sigma=-1$, and in the presence of a strong surface tension when $\sigma=1$. The equation with $\sigma=-1$ (negative dispersion) is also called the KP-II equation, whereas the one with $\sigma=1$ (positive dispersion) is called the KP-I equation. It turns out that the stability properties of the line solitary waves strongly depend upon the sign of $\sigma$ : when $\sigma=-1$ the line solitary waves are transversely stable, and when $\sigma=-1$ they are unstable $[1,10]$.

In addition to the well-known solitary waves, the $\mathrm{KdV}$ equation possesses a family of periodic traveling waves. In this paper we are interested in the stability of these periodic waves as solutions of the KP equation, i.e., their transverse stability. As solutions of the KdV equation, the stability of these periodic traveling waves is quite well-understood, though the results are less complete than those for solitary waves. In contrast to the case of localized solutions, for periodic waves of dispersive equations there are mainly to types of stability results: nonlinear (orbital) stability with respect to periodic perturbations which have the same period as the traveling wave, and spectral stability with respect to non-periodic (localized or bounded) perturbations. The question of nonlinear stability with respect to non-periodic perturbations is widely open, so far. For the KdV, it has been recently shown 
that the periodic traveling waves are orbitally stable with respect to periodic perturbations [2], and spectrally stable with respect to non-periodic perturbations $[3,7]$.

Very recently, Johnson and Zumbrun [9] derived an instability criterion for the transverse instability of periodic waves for the generalized KP equation, with respect to perturbations which are periodic in the direction of propagation and have long wavelengths in the transverse direction. In contrast, we restrict here to the case of small periodic waves, and consider the question of spectral stability for more general perturbations for the KP-I equation, and for the same type of perturbations for the KP-II equation. A particularity of the KP equation is that the spectral problem cannot be directly formulated in terms of the spectrum of a linear operator, because of the mixed time-space derivative $u_{t x}$ appearing in (1.1). We present a functional set-up which leads to such a formulation, for both periodic and non-periodic perturbations. For periodic perturbations, we use the invertibility of the operator $\partial_{x}$ when restricted to a space of periodic functions with zero mean, and for non-periodic perturbations we rely upon Floquet theory for differential operators with periodic coefficients and the invertibility of the operator $\partial_{x}+i \gamma$. For the analysis of the spectra of the resulting operators we rely upon several tools from the perturbation theory for linear operators, which turn out to be suitable for the analysis of spectra of differential operators with periodic coefficients $[6,8]$. In addition, we use the decomposition of these operators in the product of a skew-adjoint with a self-adjoint operator, and a counting result which relates the number of unstable eigenvalues of the product operator to the number of negative eigenvalues of the self-adjoint operator [7]. We focus on the KP-I equation, for which we show that the small periodic waves are transversely unstable, with respect to both types of perturbations. For the KP-II equation, a complete analysis of the spectra turns out to be more difficult, because of the different behavior of the dispersion relation. Restricting to perturbations which are periodic in the direction of propagation and have long wavelengths in the transverse direction, we show that the small periodic waves are spectrally stable with respect to such perturbations. In particular, these results show that the periodic waves have the same stability properties as the solitary waves, but under the restriction to long wavelength transverse perturbations for the KP-II equation.

The paper is organized as follows. In Section 2, we describe the family of the one-dimensional periodic traveling waves of the KP equation and give a parameterization of the small waves. The spectral stability problem is first formulated in Section 3, and then reformulated for periodic perturbations in Section 4.1, and for non-periodic perturbations in Section 5.1. The results in these latter two sections are presented for the KP-I equation, but it is easily seen that the same results hold for the KP-II equation. We discuss the spectra of the resulting linear operators, and show the transverse spectral instability of the small periodic waves of the KP-I equation for periodic perturbations in Section 4, and for non-periodic perturbations in Section 5. In Section 6, we prove the transverse spectral stability result for the periodic waves of the KP-II equation mentioned above, and we conclude with a brief discussion in Section 7.

\section{One-dimensional periodic traveling waves}

One-dimensional traveling waves of the KP equation (1.1) are solutions of the form

$$
u(x, y, t)=v(x-c t),
$$


where $c \in \mathbb{R}$ is the speed of propagation, and $v$ satisfies the ODE

$$
\left(c v^{\prime}+v^{\prime \prime \prime}+v v^{\prime}\right)^{\prime}=0 .
$$

Integrating this equation twice, and writing $x$ instead of $x-c t$, we obtain the second order ODE

$$
v^{\prime \prime}=-c v-\frac{1}{2} v^{2}+b+d x,
$$

in which $b$ and $d$ are arbitrary constants. Since we are interested in periodic solutions, we can set $d=0$ and the equation becomes

$$
v^{\prime \prime}=-c v-\frac{1}{2} v^{2}+b
$$

Symmetries The KP equation (1.1) possesses the Galilean invariance

$$
u(x, y, t) \mapsto u(x+\alpha t, y, t)+\alpha,
$$

which leads to the invariance of the traveling-wave equation (2.1) under the transformation

$$
v \mapsto v+\alpha, \quad c \rightarrow c-\alpha, \quad b \mapsto b+\alpha c-\frac{1}{2} \alpha^{2} .
$$

As a consequence, we can restrict to positive speeds $c>0$, and to $b=0$. In addition, the KP equation (1.1) possesses the scaling invariance

$$
u(x, y, t) \mapsto c u(\sqrt{c} x, c y, c \sqrt{c} t)
$$

which allows to restrict to the speed $c=1$.

Small periodic waves Restricting to $c=1$ and $b=0$, the equation (2.1) becomes

$$
v^{\prime \prime}=-v-\frac{1}{2} v^{2}
$$

In the phase-plane $\left(v, v^{\prime}\right)$, this equation possesses the equilibria $(0,0)$ and $(-2,0)$, which are a center and a saddle, respectively. The center $(0,0)$ is surrounded by a one-parameter family of periodic orbits, with periods $L \in(2 \pi, \infty)$. As $L \rightarrow 2 \pi$, the periodic orbits shrink to the equilibrium $(0,0)$, whereas for $L \rightarrow \infty$ the periodic orbits tend to a homoclinic orbit connecting the saddle equilibrium $(-2,0)$ to itself. These periodic orbits are symmetric with respect to the $v$-axis in the phase-plane $\left(v, v^{\prime}\right)$, so that to each periodic orbit corresponds a periodic solution of $(2.2)$ which is even in $x$, and unique up to translations in $x$.

We are interested here in the periodic orbits close to the center equilibrium $(0,0)$, which correspond to small periodic traveling waves of the KP equation (1.1). Looking for solutions of (2.2) of the form $v(x)=P(k x)$, where $k$ is the wavenumber and $P$ is a $2 \pi$-periodic function, a direct calculation shows that the one-parameter family of small periodic solutions of $(2.2)$, which are even in $x$, is given by

$$
v_{a}(x)=P_{a}\left(k_{a} x\right)
$$

where

$$
P_{a}(z)=a \cos (z)+\frac{1}{4}\left(\frac{1}{3} \cos (2 z)-1\right) a^{2}+O\left(|a|^{3}\right), \quad k_{a}^{2}=1-\frac{5}{24} a^{2}+O\left(a^{4}\right) .
$$


The parameter $a$ is small and corresponds to the Fourier mode 1 in the Fourier expansion of the $2 \pi$-periodic function $P_{a}$,

$$
a=\frac{1}{\pi} \int_{0}^{2 \pi} P_{a}(z) \cos (z) d z .
$$

The function $P_{a}$ is even in $z$, and notice the symmetry

$$
P_{a}(z+\pi)=P_{-a}(z), \quad k_{a}=k_{-a} .
$$

Taking into account the translation invariance we obtain a two-parameter family of periodic solutions of (2.2), and including the parameters $b$ and $c$, we find a four parameter family of one-dimensional periodic traveling waves of the KP equation (1.1).

\section{The spectral stability problem}

Consider the one-dimensional periodic traveling waves of the KP equation (1.1) found in Section 2,

$$
u_{a}(x, y, t)=P_{a}\left(k_{a}(x-t)\right) .
$$

We introduce the scaling

$$
z=k_{a}(x-t), \quad \tilde{y}=k_{a}^{2} y, \quad \tilde{t}=k_{a}^{3} t
$$

which transforms the KP equation (1.1) into

$$
u_{t z}-u_{z z z z}-\frac{1}{k_{a}^{2}} u_{z z}-\frac{1}{k_{a}^{2}}\left(u u_{z}\right)_{z}+\sigma u_{y y}=0,
$$

where we have dropped the tilde for notational simplicity. Then $P_{a}$ is a stationary solution of (3.1), and our goal is to study its spectral stability.

Following the standard approach to spectral stability, we linearize (3.1) about the stationary solution $P_{a}$ and obtain the linear evolution equation

$$
w_{t z}-w_{z z z z}-\frac{1}{k_{a}^{2}} w_{z z}-\frac{1}{k_{a}^{2}}\left(P_{a} w\right)_{z z}+\sigma w_{y y}=0 .
$$

This equation has coefficients depending upon $z$, only, so that we make the Ansatz,

$$
w(z, y, t)=e^{\lambda t+i \ell y} W(z),
$$

which leads to the equation

$$
\lambda W_{z}-W_{z z z z}-\frac{1}{k_{a}^{2}} W_{z z}-\frac{1}{k_{a}^{2}}\left(P_{a} W\right)_{z z}-\sigma \ell^{2} W=0 .
$$

The left hand side of this equation defines the linear differential operator

$$
\mathcal{M}_{a}(\lambda, \ell)=\lambda \partial_{z}-\partial_{z}^{4}-\frac{1}{k_{a}^{2}} \partial_{z}^{2}\left(\left(1+P_{a}\right) \cdot\right)-\sigma \ell^{2}
$$

and the spectral stability problem is concerned with the invertibility of this operator for $\lambda \in \mathbb{C}$ and $\ell \in \mathbb{R}$ : the periodic wave is spectrally stable if this operator is invertible for any $\lambda \in \mathbb{C}$ with $\operatorname{Re} \lambda>0$, and unstable otherwise. The type of the allowed perturbations is determined by the choice of the function space and the values of $\ell$. 
One-dimensional perturbations The spectral stability problem for one-dimensional perturbations, i.e., perturbations which are independent of $y$, corresponds to the case $\ell=0$, when

$$
\mathcal{M}_{a}(\lambda, 0)=\partial_{z} \mathcal{K}_{a}(\lambda), \quad \mathcal{K}_{a}(\lambda)=\lambda-\partial_{z}^{3}-\frac{1}{k_{a}^{2}} \partial_{z}\left(\left(1+P_{a}\right) \cdot\right) .
$$

The linear operator $\mathcal{K}_{a}(\lambda)$ in this decomposition is in fact the linear operator arising in the spectral stability problem for the $\mathrm{KdV}$ equation (1.2). As solutions of the KdV equation, the periodic waves $P_{a}$ are spectrally stable with respect to perturbations which are periodic in $z$, but also with respect to perturbations which are localized or bounded in $z$, i.e., the linear operator $\mathcal{K}_{a}(\lambda)$ is invertible, for any $\lambda \in \mathbb{C}, \operatorname{Re} \lambda>0$, in $L^{2}(0,2 \pi)$, and also $L^{2}(\mathbb{R})$ or $C_{b}(\mathbb{R})$ (e.g., see $\left.[7,3]\right)$. On the other hand, the operator $\partial_{z}$ is not invertible in these spaces, so that the linear operator $\mathcal{M}_{a}(\lambda, 0)$ is not invertible, for any $\lambda \in \mathbb{C}$, $\operatorname{Re} \lambda>0$. This ill-posedness of the spectral stability problem for one-dimensional perturbations shows that for such perturbations we have to use the operator $\mathcal{K}_{a}(\lambda)$ instead of $\mathcal{M}_{a}(\lambda, 0)$, i.e., the KP operator $\mathcal{M}_{a}(\lambda, \ell)$ should be replaced by the $\mathrm{KdV}$ operator $\mathcal{K}_{a}(\lambda)$, when $\ell=0$.

Two-dimensional perturbations Truly two-dimensional perturbations correspond to values $\ell \neq 0$. We consider three types of such perturbations: perturbations which are periodic in $z$, perturbations which are localized in $z$, and perturbations which are bounded in $z$. The type of these perturbations is determined by choice of the function space in which acts the linear operator $\mathcal{M}_{a}(\lambda, \ell)$ :

(i) $\mathcal{M}_{a}(\lambda, \ell)$ is considered in $L^{2}(0,2 \pi)$, with domain

$$
H_{\mathrm{per}}^{4}(0,2 \pi)=\left\{f \in H_{\mathrm{loc}}^{4}(\mathbb{R}) ; f(z)=f(z+2 \pi), \forall z \in \mathbb{R}\right\},
$$

for perturbations which are periodic in $z$;

(ii) $\mathcal{M}_{a}(\lambda, \ell)$ is considered in $L^{2}(\mathbb{R})$, with domain $H^{4}(\mathbb{R})$, for perturbations which are localized in $z$;

(iii) $\mathcal{M}_{a}(\lambda, \ell)$ is considered in $C_{b}(\mathbb{R})$, with domain $C_{b}^{4}(\mathbb{R})$, for perturbations which are bounded in $z$.

Spectral stability Summarizing, we give the following definition.

Definition 3.1 (Spectral stability) (i) We say that the periodic wave $P_{a}$ is spectrally stable in one dimension with respect to periodic perturbations (resp. localized or bounded perturbations), if the $K d V$ operator $\mathcal{K}_{a}(\lambda)$ acting in $L^{2}(0,2 \pi)$ (resp. $L^{2}(\mathbb{R})$ or $C_{b}(\mathbb{R})$ ) with domain $H_{\text {per }}^{3}(0,2 \pi)$ (resp. $H^{3}(\mathbb{R})$ or $C_{b}^{3}(\mathbb{R})$ ) is invertible, for any $\lambda \in \mathbb{C}, \operatorname{Re} \lambda>0$.

(ii) We say that the periodic wave $P_{a}$ is transversely spectrally stable with respect to periodic perturbations (resp. localized or bounded perturbations), if it is spectrally stable in one dimension, and the KP operator $\mathcal{M}_{a}(\lambda, \ell)$ acting in $L^{2}(0,2 \pi)$ (resp. $L^{2}(\mathbb{R})$ or $C_{b}(\mathbb{R})$ ) with domain $H_{\text {per }}^{4}(0,2 \pi)$ (resp. $H^{4}(\mathbb{R})$ or $C_{b}^{4}(\mathbb{R})$ ) is invertible, for any $\lambda \in \mathbb{C}, \operatorname{Re} \lambda>0$ and any $\ell \neq 0$;

The results in $[7,3]$ show that the periodic waves $P_{a}$ are spectrally stable in one dimension. We discuss their transverse spectral stability in the following sections. It turns out that the stability properties strongly depend upon the sign of $\sigma$. We focus on the case $\sigma=1$, i.e., the KP-I equation, in Sections 4 and 5 , and then briefly discuss the case $\sigma=-1$, i.e., the KP-II equation, in Section 6 . Notice that 
depending upon the values of $\ell$ we may distinguish three different regimes: short wavelength transverse perturbations, when $\ell \gg 1$, long wavelength transverse perturbations, when $\ell \ll 1$, and finite wavelength transverse perturbations, otherwise. The results in [9] concern the transverse spectral stability with respect to periodic perturbations in the regime of long wavelength transverse perturbations.

\section{KP-I equation: periodic perturbations}

In this section we study the transverse spectral stability of the periodic waves $P_{a}$ with respect to periodic perturbations, for the KP-I equation, i.e., when $\sigma=1$. More precisely, we study the invertibility of the operator

$$
\mathcal{M}_{a}(\lambda, \ell)=\lambda \partial_{z}-\partial_{z}^{4}-\frac{1}{k_{a}^{2}} \partial_{z}^{2}\left(\left(1+P_{a}\right) \cdot\right)-\ell^{2},
$$

acting in $L^{2}(0,2 \pi)$ with domain $H_{\text {per }}^{4}(0,2 \pi)$, for $\lambda \in \mathbb{C}, \operatorname{Re} \lambda>0$, and $\ell \in \mathbb{R}, \ell \neq 0$.

\subsection{Reformulation of the spectral stability problem}

We show that the question of the invertibility of the operator $\mathcal{M}_{a}(\lambda, \ell)$ is equivalent to the study of the spectrum of the linear operator

$$
\mathcal{A}_{a}(\ell)=\partial_{z}^{3}+\frac{1}{k_{a}^{2}} \partial_{z}\left(\left(1+P_{a}\right) \cdot\right)+\ell^{2} \partial_{z}^{-1}
$$

acting in the space

$$
L_{0}^{2}(0,2 \pi)=\left\{f \in L^{2}(0,2 \pi) ; \int_{0}^{2 \pi} f(z) d z=0\right\}
$$

of square-integrable functions on $(0,2 \pi)$ with of zero-mean, with domain $H_{\text {per }}^{3}(0,2 \pi) \cap L_{0}^{2}(0,2 \pi)$. Here $\partial_{z}^{-1}$ is the inverse of the restriction of $\partial_{z}$ to the subspace $L_{0}^{2}(0,2 \pi)$.

Lemma 4.1 Assume that $\lambda \in \mathbb{C}$ and $\ell \in \mathbb{R}, \ell \neq 0$. Then the linear operator $\mathcal{M}_{a}(\lambda, \ell)$ acting in $L^{2}(0,2 \pi)$ with domain $H_{\mathrm{per}}^{4}(0,2 \pi)$ is invertible if and only if its restriction to the subspace $L_{0}^{2}(0,2 \pi)$ is an invertible operator.

Proof. First, notice that the subspace $L_{0}^{2}(0,2 \pi) \subset L^{2}(0,2 \pi)$ is invariant under the action of $\mathcal{M}_{a}(\lambda, \ell)$, since

$$
\int_{0}^{2 \pi}\left(\mathcal{M}_{a}(\lambda, \ell) W\right)(z) d z=-\ell^{2} \int_{0}^{2 \pi} W(z) d z=0
$$

for any $W \in H_{\text {per }}^{4}(0,2 \pi) \cap L_{0}^{2}(0,2 \pi)$. Next, the operator $\mathcal{M}_{a}(\lambda, \ell)$ has compact resolvent, since $H_{\text {per }}^{4}(0,2 \pi)$ is compactly embedded in $L^{2}(0,2 \pi)$. Consequently, the spectrum of $\mathcal{M}_{a}(\lambda, \ell)$ consists of isolated eigenvalues with finite algebraic multiplicity. In particular, $\mathcal{M}_{a}(\lambda, \ell)$ is invertible if and only if 0 is an eigenvalue of $\mathcal{M}_{a}(\lambda, \ell)$, i.e., if and only if there exists $W \in H_{\text {per }}^{4}(0,2 \pi), W \neq 0$, such that

$$
\mathcal{M}_{a}(\lambda, \ell) W=0
$$

Since $\ell \neq 0$, we conclude that $W \in L_{0}^{2}(0,2 \pi)$, so that any eigenfunction $W \in H_{\text {per }}^{4}(0,2 \pi)$ associated to the eigenvalue 0 belongs to $L_{0}^{2}(0,2 \pi)$. This implies that 0 is an eigenvalue of $\mathcal{M}_{a}(\lambda, \ell)$ if and only if 0 is an eigenvalue of the restriction of $\mathcal{M}_{a}(\lambda, \ell)$ to $L_{0}^{2}(0,2 \pi)$, which proves the lemma. 
Since the operator $\partial_{z}$ acting in $L_{0}^{2}(0,2 \pi)$ with domain $H_{\text {per }}^{1}(0,2 \pi) \cap L_{0}^{2}(0,2 \pi)$ is invertible, the following result is an immediate consequence of the above lemma.

Corollary 4.2 Assume that $\lambda \in \mathbb{C}$ and $\ell \in \mathbb{R}, \ell \neq 0$. Then the linear operator $\mathcal{M}_{a}(\lambda, \ell)$ acting in $L^{2}(0,2 \pi)$ with domain $H_{\mathrm{per}}^{4}(0,2 \pi)$ is invertible if and only if $\lambda$ belongs to the spectrum of the operator $\mathcal{A}_{a}(\ell)$ acting in $L_{0}^{2}(0,2 \pi)$ with domain $H_{\mathrm{per}}^{3}(0,2 \pi) \cap L_{0}^{2}(0,2 \pi)$.

Our problem consists now in the study of the spectrum of the operator $\mathcal{A}_{a}(\ell)$ acting in $L_{0}^{2}(0,2 \pi)$ with domain $H_{\text {per }}^{3}(0,2 \pi) \cap L_{0}^{2}(0,2 \pi)$, for $\ell \in \mathbb{R}, \ell \neq 0$. This operator has compact resolvent, just as $\mathcal{M}_{a}(\lambda, \ell)$, so that its spectrum consists of isolated eigenvalues with finite algebraic multiplicity. In addition, the spectrum has the following symmetry property.

Lemma 4.3 The spectrum of $\mathcal{A}_{a}(\ell)$ is symmetric with respect to both the real and the imaginary axis.

Proof. First, the spectrum of $\mathcal{A}_{a}(\ell)$ is symmetric with respect to the real axis, since $\mathcal{A}_{a}(\ell)$ is a real operator. Next, consider the reflection $\mathcal{S}$ defined by

$$
\mathcal{S} W(z)=W(-z),
$$

and notice that $\mathcal{A}_{a}(\ell)$ anti-commutes with $\mathcal{S}$,

$$
\left(\mathcal{A}_{a}(\ell) \mathcal{S} W\right)(z)=\mathcal{A}_{a}(\ell)(W(-z))=-\left(\mathcal{A}_{a}(\ell) W\right)(-z)=-\left(\mathcal{S A}_{a}(\ell) W\right)(z),
$$

where we have used the fact that $P_{a}$ is an even function. If $\lambda$ is an eigenvalue of $\mathcal{A}_{a}(\ell)$ with associated eigenvector $W_{\lambda}$,

$$
\mathcal{A}_{a}(\ell) W_{\lambda}=\lambda W_{\lambda}
$$

then

$$
\mathcal{A}_{a}(\ell) \mathcal{S} W_{\lambda}=-\mathcal{S} \mathcal{A}_{a}(\ell) W_{\lambda}=-\lambda \mathcal{S} W_{\lambda}
$$

Consequently, $-\lambda$ is an eigenvalue of $\mathcal{A}_{a}(\ell)$. This implies that the spectrum of $\mathcal{A}_{a}(\ell)$ is also symmetric with respect to the origin, and completes the proof.

The analysis of the spectrum of $\mathcal{A}_{a}(\ell)$ in the next sections relies upon two main tools:

- the decomposition of $\mathcal{A}_{a}(\ell)$ into the product of a skew-adjoint and a self-adjoint operator:

$$
\mathcal{A}_{a}(\ell)=-\partial_{z} \mathcal{L}_{a}(\ell), \quad \mathcal{L}_{a}(\ell)=-\partial_{z}^{2}-\frac{1}{k_{a}^{2}}\left(\left(1+P_{a}\right) \cdot\right)-\ell^{2} \partial_{z}^{-2},
$$

which leads to a simple characterization of the unstable eigenvalues of $\mathcal{A}_{a}(\ell)$;

- perturbation arguments for linear operators: we regard $\mathcal{A}_{a}(\ell)$ as a perturbation, for small $a$, of the operator with constant coefficients

$$
\mathcal{A}_{0}(\ell)=\partial_{z}^{3}+\partial_{z}+\ell^{2} \partial_{z}^{-1}
$$

obtained by setting $a=0$. 
The main result is the following theorem showing the spectral instability of the periodic waves $P_{a}$, for

a sufficiently small. The instability is due to a pair of real eigenvalues, with opposite signs, which is found in the long wavelength regime, for $\ell^{2}=O\left(a^{2}\right)$.

Theorem 1 For any a sufficiently small, there exists $\ell_{a}^{2}=\frac{1}{12} a^{2}+O\left(a^{4}\right)$, such that

(i) for any $\ell^{2} \geqslant \ell_{a}^{2}$, the spectrum of $\mathcal{A}_{a}(\ell)$ is purely imaginary;

(ii) for any $\ell^{2}<\ell_{a}^{2}$, the spectrum of $\mathcal{A}_{a}(\ell)$ is purely imaginary, except for a pair of simple real eigenvalues, with opposite signs.

\subsection{Characterization of the unstable spectrum}

Consider the decomposition (4.2) of the linear operator $\mathcal{A}_{a}(\ell)$. The operators $-\partial_{z}$ and $\mathcal{L}_{a}(\ell)$ in this decomposition are skew-adjoint and self-adjoint, respectively, as operators acting in $L^{2}(0,2 \pi)$. In [7] it has been shown, under some general assumptions, that for an operator $\mathcal{A}=\mathcal{J} \mathcal{L}$, where $\mathcal{J}$ and $\mathcal{L}$ are skew-adjoint and self-adjoint, respectively, the number of unstable eigenvalues, counted with multiplicities, is not larger than the number of negative eigenvalues of the operator $\mathcal{L}$. In particular, if $\mathcal{L}$ has no negative eigenvalues, then $\mathcal{A}$ has no unstable eigenvalues. For the operators in (4.2) we cannot directly apply this result, since the subspace $L_{0}^{2}(0,2 \pi)$ on which acts $\mathcal{A}_{a}(\ell)$ is not an invariant subspace for the operator $\mathcal{L}_{a}(\ell)$. Nevertheless, we can use part of the arguments in [7] to prove the following result.

Lemma 4.4 Assume that $\lambda$ is an eigenvalue of the operator $\mathcal{A}_{a}(\ell)$ acting in $L_{0}^{2}(0,2 \pi)$, and that $W_{\lambda}$ is an associated eigenvector. If $\operatorname{Re} \lambda \neq 0$, then

$$
\left\langle\mathcal{L}_{a}(\ell) W_{\lambda}, W_{\lambda}\right\rangle=0
$$

where $\langle\cdot, \cdot\rangle$ is the usual scalar product in $L^{2}(0,2 \pi)$.

Proof. Recall that the operator $\partial_{z}$ acting on $L_{0}^{2}(0,2 \pi)$ is skew-adjoint and invertible. Then, following the proof of [7, Lemma 2.7], we find

$$
\left\langle\mathcal{L}_{a}(\ell) W_{\lambda}, W_{\lambda}\right\rangle=\left\langle\mathcal{L}_{a}(\ell) W_{\lambda}, \partial_{z} \partial_{z}^{-1} W_{\lambda}\right\rangle=\left\langle\mathcal{A}_{a}(\ell) W_{\lambda}, \partial_{z}^{-1} W_{\lambda}\right\rangle=\lambda\left\langle W_{\lambda}, \partial_{z}^{-1} W_{\lambda}\right\rangle,
$$

and similarly,

$$
\left\langle W_{\lambda}, \mathcal{L}_{a}(\ell) W_{\lambda}\right\rangle=\left\langle\partial_{z} \partial_{z}^{-1} W_{\lambda}, \mathcal{L}_{a}(\ell) W_{\lambda}\right\rangle=\left\langle\partial_{z}^{-1} W_{\lambda}, \mathcal{A}_{a}(\ell) W_{\lambda}\right\rangle=\bar{\lambda}\left\langle\partial_{z}^{-1} W_{\lambda}, W_{\lambda}\right\rangle=-\bar{\lambda}\left\langle W_{\lambda}, \partial_{z}^{-1} W_{\lambda}\right\rangle .
$$

Since $\mathcal{L}_{a}(\ell)$ is self-adjoint, we conclude that

$$
(\lambda+\bar{\lambda})\left\langle\mathcal{L}_{a}(\ell) W_{\lambda}, W_{\lambda}\right\rangle=0
$$

so that $\left\langle\mathcal{L}_{a}(\ell) W_{\lambda}, W_{\lambda}\right\rangle=0$, since $\operatorname{Re} \lambda \neq 0$.

Corollary 4.5 Assume that there exists a positive constant $c$ such that

$$
\left\langle\mathcal{L}_{a}(\ell) W, W\right\rangle \geqslant c\langle W, W\rangle, \quad \forall W \in H_{\mathrm{per}}^{3}(0,2 \pi) \cap L_{0}^{2}(0,2 \pi) .
$$

Then the spectrum of $\mathcal{A}_{a}(\ell)$ is purely imaginary.

Proof. Assuming that there exists an eigenvalue $\lambda$ of $\mathcal{A}_{a}(\ell)$ with $\operatorname{Re} \lambda \neq 0$, then any associated eigenvector $W_{\lambda} \neq 0$ satisfies $\left\langle\mathcal{L}_{a}(\ell) W_{\lambda}, W_{\lambda}\right\rangle>0$, by hypothesis. This contradicts the result in Lemma 4.4, and proves the corollary. 


\subsection{Finite and short wavelength transverse perturbations}

We start the analysis of the spectrum of $\mathcal{A}_{a}(\ell)$ with the values of $\ell$ away from the origin, $|\ell| \geqslant \ell_{*}$, for some $\ell_{*}>0$, i.e., finite and short wavelength transverse perturbations. We use the result in Corollary 4.5 to show that the spectrum of $\mathcal{A}_{a}(\ell)$ is purely imaginary for such values of $\ell$, provided $a$ is sufficiently small.

Lemma 4.6 Assume that $\ell_{*}>0$. There exists $a_{*}>0$, such that the spectrum of $\mathcal{A}_{a}(\ell)$ is purely imaginary, for any $\ell$ and $a$ satisfying $|\ell| \geqslant \ell_{*}$ and $|a| \leqslant a_{*}$.

Proof. According to the Corollary 4.5 it is enough to show that

$$
\left\langle\mathcal{L}_{a}(\ell) W, W\right\rangle \geqslant c_{*}\langle W, W\rangle, \quad \forall W \in H_{\text {per }}^{3}(0,2 \pi) \cap L_{0}^{2}(0,2 \pi),
$$

for some $c_{*}>0$. We write

$$
\mathcal{L}_{a}(\ell)=\mathcal{L}_{0}(\ell)+\widetilde{\mathcal{L}}_{a}, \quad \mathcal{L}_{0}(\ell)=-\partial_{z}^{2}-1-\ell^{2} \partial_{z}^{-2}, \quad \widetilde{\mathcal{L}}_{a}=1-\frac{1}{k_{a}^{2}}\left(1+P_{a}\right) .
$$

The operator $\mathcal{L}_{0}(\ell)$ has constant coefficients, and using Fourier series we find that its spectrum in $L_{0}^{2}(0,2 \pi)$ is given by

$$
\sigma\left(\mathcal{L}_{0}(\ell)\right)=\left\{\mu_{n}(\ell)=n^{2}-1+\frac{\ell^{2}}{n^{2}} ; n \in \mathbb{Z}^{*}\right\}
$$

and satisfies

$$
\sigma\left(\mathcal{L}_{0}(\ell)\right) \subset\left[c_{0}, \infty\right), \quad c_{0}=\min \left\{\ell_{*}^{2}, 1\right\},
$$

for any $\ell,|\ell| \geqslant \ell_{*}$. As a consequence, we have that

$$
\left\langle\mathcal{L}_{0}(\ell) W, W\right\rangle \geqslant c_{0}\langle W, W\rangle, \quad \forall W \in H_{\text {per }}^{3}(0,2 \pi) \cap L_{0}^{2}(0,2 \pi) .
$$

The linear operator $\widetilde{\mathcal{L}}_{a}$ is bounded in $L^{2}(0,2 \pi)$, and a direct calculation shows that

$$
\left\|\widetilde{\mathcal{L}}_{a}\right\| \leqslant c_{1} a
$$

for some $c_{1}>0$, and any $a$ sufficiently small. Together with (4.5), this implies that

$$
\left\langle\mathcal{L}_{a}(\ell) W, W\right\rangle=\left\langle\mathcal{L}_{0}(\ell) W, W\right\rangle+\left\langle\widetilde{\mathcal{L}}_{a} W, W\right\rangle \geqslant\left(c_{0}-c_{1} a\right)\langle W, W\rangle, \quad \forall W \in H_{\mathrm{per}}^{3}(0,2 \pi) \cap L_{0}^{2}(0,2 \pi),
$$

which proves (4.4), for any $\ell,|\ell| \geqslant \ell_{*}$, provided $a$ is sufficiently small.

\subsection{Long wavelength transverse perturbations}

We consider now the spectrum of $\mathcal{A}_{a}(\ell)$ for small $\ell$. Notice that the arguments in the proof of Lemma 4.6 do not work when $\ell$ is small, since the constant $c_{0}$ in (4.5) tends to zero, as $\ell \rightarrow 0$.

Here, we regard $\mathcal{A}_{a}(\ell)$ as a perturbation of the operator with constant coefficients

$$
\mathcal{A}_{0}(0)=\partial_{z}^{3}+\partial_{z}
$$

acting in $L_{0}^{2}(0,2 \pi)$. A direct calculation shows that the spectrum of $\mathcal{A}_{0}(0)$ is given by

$$
\sigma\left(\mathcal{A}_{0}(0)\right)=\left\{i \omega_{n}=-i n^{3}+i n ; n \in \mathbb{Z}^{*}\right\} .
$$

In particular, zero is a double eigenvalue of $\mathcal{A}_{0}(0)$, and the remaining eigenvalues are all simple, purely imaginary, and located outside the open ball $B(0 ; 5)$ of radius 5 centered at the origin. 
Lemma 4.7 The following properties hold, for any $\ell$ and a sufficiently small.

(i) The spectrum of $\mathcal{A}_{a}(\ell)$ decomposes as

$$
\sigma\left(\mathcal{A}_{a}(\ell)\right)=\sigma_{0}\left(\mathcal{A}_{a}(\ell)\right) \cup \sigma_{1}\left(\mathcal{A}_{a}(\ell)\right)
$$

with

$$
\sigma_{0}\left(\mathcal{A}_{a}(\ell)\right) \subset B(0 ; 1), \quad \sigma_{1}\left(\mathcal{A}_{a}(\ell)\right) \subset \mathbb{C} \backslash \overline{B(0 ; 4)}
$$

(ii) The spectral projection $\Pi_{a}(\ell)$ associated with $\sigma_{0}\left(\mathcal{A}_{a}(\ell)\right)$ satisfies $\left\|\Pi_{a}(\ell)-\Pi_{0}(0)\right\|=O\left(\ell^{2}+|a|\right)$.

(iii) The spectral subspace $\mathcal{X}_{a}(\ell)=\Pi_{a}(\ell)\left(L_{0}^{2}(0,2 \pi)\right)$ is two dimensional.

Proof. (i) Consider $\lambda \in \overline{B(0 ; 4)} \backslash B(0 ; 1)$. Then $\lambda$ belongs to the resolvent set of $\mathcal{A}_{0}(0)$, i.e., the operator $\lambda-\mathcal{A}_{0}(0)$ is invertible. We write

$$
\lambda-\mathcal{A}_{a}(\ell)=\left(I-\widetilde{\mathcal{A}}_{a}(\ell)\left(\lambda-\mathcal{A}_{0}(0)\right)^{-1}\right)\left(\lambda-\mathcal{A}_{0}(0)\right),
$$

where $\widetilde{\mathcal{A}}_{a}(\ell)=\mathcal{A}_{a}(\ell)-\mathcal{A}_{0}(0)$. A straightforward calculation shows that there exist positive constants $c_{0}$ and $c_{1}$ such that

$$
\left\|\left(\lambda-\mathcal{A}_{0}(0)\right)^{-1}\right\|_{L^{2} \rightarrow H^{1}} \leqslant c_{0}, \quad\left\|\widetilde{\mathcal{A}}_{a}(\ell)\right\|_{H^{1} \rightarrow L^{2}} \leqslant c_{1}\left(\ell^{2}+|a|\right),
$$

for any $\lambda \in \overline{B(0 ; 4)} \backslash B(0 ; 1)$, and any $\ell$ and $a$ sufficiently small. Consequently,

$$
\left\|\widetilde{\mathcal{A}}_{a}(\ell)\left(\lambda-\mathcal{A}_{0}(0)\right)^{-1}\right\| \leqslant c_{0} c_{1}\left(\ell^{2}+|a|\right) \leqslant \frac{1}{2}
$$

for any $\lambda \in \overline{B(0 ; 4)} \backslash B(0 ; 1)$, provided $\ell$ and $a$ are sufficiently small, so that the operator $I-\widetilde{\mathcal{A}}_{a}(\ell)(\lambda-$ $\left.\mathcal{A}_{0}(0)\right)^{-1}$ is invertible. Consequently, $\lambda-\mathcal{A}_{a}(\ell)$ is invertible, for any $\lambda \in \overline{B(0 ; 4)} \backslash B(0 ; 1)$, which proves the first part of the lemma.

(ii) The spectral projection $\Pi_{a}(\ell)$ can be computed with the help of the Dunford integral formula

$$
\Pi_{a}(\ell)=\frac{1}{2 \pi i} \int_{\partial B(0 ; 1)}\left(\lambda-\mathcal{A}_{a}(\ell)\right)^{-1} d \lambda
$$

where, according to the previous arguments,

$$
\begin{aligned}
\left(\lambda-\mathcal{A}_{a}(\ell)\right)^{-1} & =\left(\lambda-\mathcal{A}_{0}(0)\right)^{-1}\left(I-\widetilde{\mathcal{A}}_{a}(\ell)\left(\lambda-\mathcal{A}_{0}(0)\right)^{-1}\right)^{-1} \\
& =\left(\lambda-\mathcal{A}_{0}(0)\right)^{-1} \sum_{k \geqslant 0}\left(\widetilde{\mathcal{A}}_{a}(\ell)\left(\lambda-\mathcal{A}_{0}(0)\right)^{-1}\right)^{k} \\
& =\left(\lambda-\mathcal{A}_{0}(0)\right)^{-1}+\left(\lambda-\mathcal{A}_{0}(0)\right)^{-1} \sum_{k \geqslant 1}\left(\widetilde{\mathcal{A}}_{a}(\ell)\left(\lambda-\mathcal{A}_{0}(0)\right)^{-1}\right)^{k} .
\end{aligned}
$$

Consequently,

$$
\Pi_{a}(\ell)-\Pi_{0}(0)=\frac{1}{2 \pi i} \int_{\partial B(0 ; 1)}\left(\lambda-\mathcal{A}_{0}(0)\right)^{-1} \sum_{k \geqslant 1}\left(\widetilde{\mathcal{A}}_{a}(\ell)\left(\lambda-\mathcal{A}_{0}(0)\right)^{-1}\right)^{k} d \lambda
$$


and since

$$
\left\|\left(\lambda-\mathcal{A}_{0}(0)\right)^{-1} \sum_{k \geqslant 1}\left(\widetilde{\mathcal{A}}_{a}(\ell)\left(\lambda-\mathcal{A}_{0}(0)\right)^{-1}\right)^{k}\right\| \leqslant 2 c_{0}^{2} c_{1}\left(\ell^{2}+|a|\right),
$$

for any $\lambda \in \partial B(0 ; 1)$, and any $\ell$ and $a$ sufficiently small, we conclude that $\left\|\Pi_{a}(\ell)-\Pi_{0}(0)\right\|=O\left(\ell^{2}+|a|\right)$.

(iii) The last part of the lemma is an immediate consequence of the estimate in (ii) (e.g., see [8, Lemma B.1]).

Next, we use the result in Lemma 4.4, to locate the eigenvalues in $\sigma_{1}\left(\mathcal{A}_{a}(\ell)\right)$, and then we determine the two eigenvalues in $\sigma_{0}\left(\mathcal{A}_{a}(\ell)\right)$, by computing an expansion of these eigenvalues for small $\ell$ and $a$. These results complete the proof of Theorem 1.

Lemma 4.8 Consider the decomposition of the spectrum of $\mathcal{A}_{a}(\ell)$ in Lemma 4.7. Then $\sigma_{1}\left(\mathcal{A}_{a}(\ell)\right) \subset$ $i \mathbb{R}$, for any $\ell$ and a sufficiently small.

Proof. Consider the restriction $\mathcal{A}_{a}^{1}(\ell)$ of $\mathcal{A}_{a}(\ell)$ to the spectral subspace $\mathcal{Y}_{a}(\ell)=\left(I-\Pi_{a}(\ell)\right)\left(L_{0}^{2}(0,2 \pi)\right)$, so that

$$
\sigma_{1}\left(\mathcal{A}_{a}(\ell)\right)=\sigma\left(\mathcal{A}_{a}^{1}(\ell)\right)
$$

Assume that $\lambda$ is an eigenvalue of $\mathcal{A}_{a}^{1}(\ell)$, and that $W_{\lambda} \neq 0$ is an associated eigenvector. In particular, $W_{\lambda}=\left(I-\Pi_{a}(\ell)\right) W_{\lambda}$. We use the result in Lemma 4.4 to show that $\operatorname{Re} \lambda=0$.

Recall that $\mathcal{A}_{a}(\ell)=-\partial_{z} \mathcal{L}_{a}(\ell)$, and write

$$
\mathcal{L}_{a}(\ell)=\mathcal{L}_{0}(0)+\widetilde{\mathcal{L}}_{a}(\ell), \quad \mathcal{L}_{0}(0)=-\partial_{z}^{2}-1, \quad \widetilde{\mathcal{L}}_{a}(\ell)=1-\frac{1}{k_{a}^{2}}\left(1+P_{a}\right)-\ell^{2} \partial_{z}^{-2},
$$

so that $\widetilde{\mathcal{L}}_{a}(\ell)$ is a bounded operator on $L^{2}(0,2 \pi)$, with norm

$$
\left\|\widetilde{\mathcal{L}}_{a}(\ell)\right\| \leqslant c_{0}\left(\ell^{2}+|a|\right)
$$

for some positive constant $c_{0}$, and any $\ell$ and $a$ sufficiently small. We set $\widetilde{\Pi}_{a}(\ell)=\Pi_{a}(\ell)-\Pi_{0}(0)$, and then the property in Lemma 4.7 (ii) shows that

$$
\left\|\widetilde{\Pi}_{a}(\ell)\right\|=\left\|\Pi_{a}(\ell)-\Pi_{0}(0)\right\| \leqslant c_{1}\left(\ell^{2}+|a|\right),
$$

for some positive constant $c_{1}$, and any $\ell$ and $a$ sufficiently small. We compute

$$
\begin{aligned}
\left\langle\mathcal{L}_{a}(\ell) W_{\lambda}, W_{\lambda}\right\rangle= & \left\langle\mathcal{L}_{a}(\ell)\left(I-\Pi_{a}(\ell)\right) W_{\lambda},\left(I-\Pi_{a}(\ell)\right) W_{\lambda}\right\rangle \\
= & \left\langle\mathcal{L}_{0}(0)\left(I-\Pi_{a}(\ell)\right) W_{\lambda},\left(I-\Pi_{a}(\ell)\right) W_{\lambda}\right\rangle+\left\langle\widetilde{\mathcal{L}}_{a}(\ell)\left(I-\Pi_{a}(\ell)\right) W_{\lambda},\left(I-\Pi_{a}(\ell)\right) W_{\lambda}\right\rangle \\
= & \left\langle\mathcal{L}_{0}(0)\left(I-\Pi_{0}(0)\right) W_{\lambda},\left(I-\Pi_{0}(0)\right) W_{\lambda}\right\rangle-\left\langle\mathcal{L}_{0}(0) \widetilde{\Pi}_{a}(\ell) W_{\lambda},\left(I-\Pi_{0}(0)\right) W_{\lambda}\right\rangle \\
& -\left\langle\mathcal{L}_{0}(0)\left(I-\Pi_{a}(\ell)\right) W_{\lambda}, \widetilde{\Pi}_{a}(\ell) W_{\lambda}\right\rangle+\left\langle\widetilde{\mathcal{L}}_{a}(\ell)\left(I-\Pi_{a}(\ell)\right) W_{\lambda},\left(I-\Pi_{a}(\ell)\right) W_{\lambda}\right\rangle .
\end{aligned}
$$

Since the spectrum of the restriction of $\mathcal{L}_{0}(0)$ to $\left(I-\Pi_{0}(0)\right) L_{0}^{2}(0,2 \pi)$ consists of the eigenvalues

$$
n^{2}-1, \quad n \in \mathbb{Z} \backslash\{-1,0,1\},
$$

we have that

$$
\left\langle\mathcal{L}_{0}(0)\left(I-\Pi_{0}(0)\right) W_{\lambda},\left(I-\Pi_{0}(0)\right) W_{\lambda}\right\rangle \geqslant 3\left\langle W_{\lambda}, W_{\lambda}\right\rangle,
$$


and taking into account the estimates (4.6) and (4.7), we conclude that

$$
\left\langle\mathcal{L}_{a}(\ell) W_{\lambda}, W_{\lambda}\right\rangle \geqslant\left(3-c_{2}\left(\ell^{2}+|a|\right)\right)\left\langle W_{\lambda}, W_{\lambda}\right\rangle,
$$

for some positive constant $c_{2}$, and any $\ell$ and $a$ sufficiently small. Consequently, $\left\langle\mathcal{L}_{a}(\ell) W_{\lambda}, W_{\lambda}\right\rangle>0$, provided $\ell$ and $a$ are sufficiently small, so that $\operatorname{Re} \lambda=0$, by Lemma 4.4.

Lemma 4.9 Assume that $\ell$ and a are sufficiently small. There exists $\ell_{a}^{2}=\frac{1}{12} a^{2}+O\left(a^{4}\right)$, such that the two eigenvalues in $\sigma_{0}\left(\mathcal{A}_{a}(\ell)\right)$ are purely imaginary, if $\ell^{2} \geqslant \ell_{a}^{2}$, and are real, one positive and one negative, if $\ell^{2}<\ell_{a}^{2}$.

Proof. The eigenvalues in $\sigma_{0}\left(\left(\mathcal{A}_{a}(\ell)\right)\right.$ are the eigenvalues of the restriction of $\mathcal{A}_{a}(\ell)$ to the twodimensional spectral subspace $\mathcal{X}_{a}(\ell)=\Pi_{a}(\ell)\left(L_{0}^{2}(0,2 \pi)\right)$. We determine the location of these eigenvalues by computing successively a basis of $\mathcal{X}_{a}(\ell)$, the $2 \times 2$ matrix representing the action of $\mathcal{A}_{a}(\ell)$ on this basis, and the eigenvalues of this matrix.

We start with the computation for $a=0$. Then

$$
\mathcal{A}_{0}(\ell)=\partial_{z}^{3}+\partial_{z}+\ell^{2} \partial_{z}^{-1}
$$

is an operator with constant coefficients, and

$$
\sigma_{0}\left(\mathcal{A}_{0}(\ell)\right)=\left\{-i \ell^{2}, i \ell^{2}\right\}
$$

The associated eigenvectors are $e^{i z}$ and $e^{-i z}$, and we choose

$$
\xi_{0}^{0}(\ell)=\sin (z), \quad \xi_{0}^{1}(\ell)=\cos (z),
$$

as basis of the corresponding spectral subspace. Since

$$
\mathcal{A}_{0}(\ell) \xi_{0}^{0}(\ell)=-\ell^{2} \xi_{0}^{1}(\ell), \quad \mathcal{A}_{0}(\ell) \xi_{0}^{1}(\ell)=\ell^{2} \xi_{0}^{0}(\ell),
$$

the $2 \times 2$ matrix representing the action of $\mathcal{A}_{0}(\ell)$ on this basis is given by

$$
M_{0}(\ell)=\left(\begin{array}{cc}
0 & \ell^{2} \\
-\ell^{2} & 0
\end{array}\right)
$$

Next, recall that $\mathcal{A}_{a}(\ell)$ anti-commutes with the reflection $\mathcal{S}$ defined by (4.1). Since

$$
\mathcal{S} \xi_{0}^{0}(\ell)=-\xi_{0}^{0}(\ell), \quad \mathcal{S} \xi_{0}^{1}(\ell)=\xi_{0}^{1}(\ell),
$$

the basis $\left\{\xi_{0}^{0}(\ell), \xi_{0}^{1}(\ell)\right\}$ can be extended to a basis $\left\{\xi_{a}^{0}(\ell), \xi_{a}^{1}(\ell)\right\}$, for $a \neq 0$, with the same property, i.e., such that $\xi_{a}^{0}(\ell)$ and $\xi_{a}^{1}(\ell)$ are odd and even in $z$, respectively. In this basis the $2 \times 2$ matrix representing the action of $\mathcal{A}_{a}(\ell)$ is of the form

$$
M_{a}(\ell)=\left(\begin{array}{cc}
0 & \ell^{2}+O(a) \\
-\ell^{2}+O(a) & 0
\end{array}\right) .
$$

In order to compute the terms of order $a$ we take $\ell=0$. The derivative $\partial_{z} P_{a}$ of the periodic wave always belong to the kernel of $\mathcal{A}_{a}(0)$,

$$
\mathcal{A}_{a}(0)\left(\partial_{z} P_{a}\right)=0
$$


due to the invariance of the KP equation under translations in $x$, and $\partial_{z} P_{a}$ is an odd function, with expansion

$$
\partial_{z} P_{a}(z)=-a \sin (z)-\frac{1}{6} \sin (2 z) a^{2}+O\left(|a|^{3}\right)
$$

Then we choose

$$
\xi_{a}^{0}(0)=-\frac{1}{a} \partial_{z} P_{a}(z)=\sin (z)+\frac{1}{6} \sin (2 z) a+O\left(a^{2}\right),
$$

as a first vector of the basis, which is compatible with the choice for $a=0$, and satisfies $\mathcal{A}_{a}(0) \xi_{a}^{0}(0)=0$. For the second vector we set

$$
\xi_{a}^{1}(0)=\cos (z)+\varphi(z) a+O\left(a^{2}\right)
$$

and

$$
\mathcal{A}_{a}(0) \xi_{a}^{1}(0)=\left(\alpha_{0}+\alpha_{1} a+\alpha_{2} a^{2}+O\left(|a|^{3}\right)\right) \xi_{a}^{0}(0) .
$$

Then a direct calculation gives

$$
\alpha_{0}=\alpha_{1}=0, \quad \alpha_{2}=-\frac{1}{12}, \quad \xi_{a}^{1}(0)=\cos (z)+\frac{1}{6} \cos (2 z) a+O\left(a^{2}\right),
$$

so that

$$
M_{a}(0)=\left(\begin{array}{cc}
0 & -\frac{1}{12} a^{2}+O\left(|a|^{3}\right) \\
0 & 0
\end{array}\right) .
$$

Together with the expression of $M_{0}(\ell)$, this shows that

$$
M_{a}(\ell)=\left(\begin{array}{cc}
0 & \ell^{2}-\frac{1}{12} a^{2}+O\left(|a|\left(\ell^{2}+a^{2}\right)\right) \\
-\ell^{2}+O\left(|a| \ell^{2}\right) & 0
\end{array}\right) .
$$

The two eigenvalues of $M_{a}(\ell)$, which are also the eigenvalues in $\sigma_{0}\left(\mathcal{A}_{a}(\ell)\right)$, are roots of the characteristic polynomial

$$
P(\lambda)=\lambda^{2}+\ell^{2}\left(\ell^{2}-\frac{1}{12} a^{2}\right)+O\left(|a| \ell^{2}\left(\ell^{2}+a^{2}\right)\right) .
$$

Furthermore, since $P_{a}(z+\pi)=P_{-a}(z)$, the two roots of this polynomial are the same for $a$ and $-a$, and we conclude that

$$
\lambda^{2}=-\ell^{2}\left(\ell^{2}-\frac{1}{12} a^{2}\right)+O\left(a^{2} \ell^{2}\left(\ell^{2}+a^{2}\right)\right)
$$

Consequently, for any a sufficiently small there exists a value

$$
\ell_{a}^{2}=\frac{1}{12} a^{2}+O\left(a^{4}\right)
$$

such that the two eigenvalues are purely imaginary when $\ell^{2}>\ell_{a}^{2}$, and real, with opposite signs when $\ell^{2}<\ell_{a}^{2}$. This proves the lemma.

\section{KP-I equation: non-periodic perturbations}

In this section we study the transverse spectral stability of the periodic waves $P_{a}$ of the KP-I equation, with respect to perturbations which are localized or bounded in $z$, i.e., we study the invertibility of the operator

$$
\mathcal{M}_{a}(\lambda, \ell)=\lambda \partial_{z}-\partial_{z}^{4}-\frac{1}{k_{a}^{2}} \partial_{z}^{2}\left(\left(1+P_{a}\right) \cdot\right)-\ell^{2},
$$

acting in $L^{2}(\mathbb{R})$ or $C_{b}(\mathbb{R})$, for $\lambda \in \mathbb{C}, \operatorname{Re} \lambda>0$, and $\ell \in \mathbb{R}, \ell \neq 0$. 


\subsection{Bloch-wave decomposition}

In contrast to the case of perturbations which are periodic in $z$ discussed in the previous section, for perturbations which are localized or bounded, the linear operator $\mathcal{M}_{a}(\lambda, \ell)$ acting in $L^{2}(\mathbb{R})$ or $C_{b}(\mathbb{R})$ has continuous spectrum. A convenient way to treat this situation is with the help of the so-called Bloch-wave decomposition, which leads to the following result.

Lemma 5.1 The linear operator $\mathcal{M}_{a}(\lambda, \ell)$ is invertible, in either $L^{2}(\mathbb{R})$ or $C_{b}(\mathbb{R})$, if and only if the linear operators

$$
\mathcal{M}_{a}(\lambda, \ell, \gamma)=\lambda\left(\partial_{z}+i \gamma\right)-\left(\partial_{z}+i \gamma\right)^{4}-\frac{1}{k_{a}^{2}}\left(\partial_{z}+i \gamma\right)^{2}\left(\left(1+P_{a}\right) \cdot\right)-\ell^{2}
$$

acting in $L^{2}(0,2 \pi)$ with domain $H_{\mathrm{per}}^{4}(0,2 \pi)$ are invertible, for any $\gamma \in\left(-\frac{1}{2}, \frac{1}{2}\right]$.

Proof. The proof relies upon Floquet theory. We refer, e.g., to [6, Proposition 1.1 (ii)-(iii)] for a detailed proof in similar situation.

The key property of the operators $\mathcal{M}_{a}(\lambda, \ell, \gamma)$ in this lemma is that they have only point spectrum, since they act in $L^{2}(0,2 \pi)$ with compactly embedded domain $H_{\text {per }}^{4}(0,2 \pi)$. When $\gamma=0$ we recover the case of periodic perturbations studied in the previous section, so that we restrict now to $\gamma \neq 0$. Then the operator $\partial_{z}+i \gamma$ is invertible in $L^{2}(0,2 \pi)$, property which leads to the following result.

Lemma 5.2 Assume that $\gamma \in\left(-\frac{1}{2}, \frac{1}{2}\right]$ and $\gamma \neq 0$. Then the linear operator $\mathcal{M}_{a}(\lambda, \ell, \gamma)$ is invertible in $L^{2}(0,2 \pi)$ if and only if $\lambda$ belongs to the spectrum of the operator

$$
\mathcal{A}_{a}(\ell, \gamma)=\left(\partial_{z}+i \gamma\right)^{3}+\frac{1}{k_{a}^{2}}\left(\partial_{z}+i \gamma\right)\left(\left(1+P_{a}\right) \cdot\right)+\ell^{2}\left(\partial_{z}+i \gamma\right)^{-1}
$$

acting in $L^{2}(0,2 \pi)$ with domain $H_{\mathrm{per}}^{3}(0,2 \pi)$.

We point out that the operator $\left(\partial_{z}+i \gamma\right)^{-1}$ becomes singular, as $\gamma \rightarrow 0$, so that the results found by replacing the study of the invertibility of $\mathcal{M}_{a}(\lambda, \ell, \gamma)$ by the study of the spectrum of $\mathcal{A}_{a}(\ell, \gamma)$ are not uniform for small $\gamma$, i.e., the set of $a$ for which these results are valid depends upon $\gamma$. We restrict here to this latter study which turns out to be simpler, and sufficient to detect the values of $\ell$ and $\gamma$ where instabilities occur.

As in the previous section, the linear operator $\mathcal{A}_{a}(\ell, \gamma)$ decomposes as

$$
\mathcal{A}_{a}(\ell, \gamma)=-\left(\partial_{z}+i \gamma\right) \mathcal{L}_{a}(\ell, \gamma), \quad \mathcal{L}_{a}(\ell, \gamma)=-\left(\partial_{z}+i \gamma\right)^{2}-\frac{1}{k_{a}^{2}}\left(\left(1+P_{a}\right) \cdot\right)-\ell^{2}\left(\partial_{z}+i \gamma\right)^{-2}
$$

in which $\partial_{z}+i \gamma$ and $\mathcal{L}_{a}(\ell, \gamma)$ are skew-adjoint and self-adjoint operators, respectively. As a consequence, the result in Corollary 4.5 also holds for these operators. However, in contrast to the previous section, here we can also directly apply the result in [7, Theorem 2.13] showing that the numbers $n\left(\mathcal{L}_{a}(\ell, \gamma)\right)$ of negative eigenvalues of $\mathcal{L}_{a}(\ell, \gamma)$ (counted with multiplicities) and $k_{u}\left(\mathcal{A}_{a}(\ell, \gamma)\right)$ of unstable eigenvalues of $\mathcal{A}_{a}(\ell, \gamma)$ (counted with multiplicities), satisfy the inequality

$$
k_{u}\left(\mathcal{A}_{a}(\ell, \gamma)\right) \leqslant n\left(\mathcal{L}_{a}(\ell, \gamma)\right)
$$


provided $\mathcal{L}_{a}(\ell, \gamma)$ is invertible. In fact the result in [7, Theorem 2.13] is more precise, but this inequality is enough for our purposes, since we shall only use this result in the case $n\left(\mathcal{L}_{a}(\ell, \gamma)\right)=0$. Actually, in this situation the inequality (5.2) also follows from Corollary 4.5. Indeed, if $n\left(\mathcal{L}_{a}(\ell, \gamma)\right)=0$ and $\mathcal{L}_{a}(\ell, \gamma)$ is invertible, then the spectrum of $\mathcal{L}_{a}(\ell, \gamma)$ is strictly positive. Since $\mathcal{L}_{a}(\ell, \gamma)$ is self-adjoint this implies the inequality (4.3), so that the spectrum of $\mathcal{A}_{a}(\ell, \gamma)$ is purely imaginary. Consequently, $k_{u}\left(\mathcal{A}_{a}(\ell, \gamma)\right)=0=n\left(\mathcal{L}_{a}(\ell, \gamma)\right)$, which proves the inequality.

Another consequence of the decomposition (5.1) is that the spectrum of $\mathcal{A}_{a}(\ell, \gamma)$ is symmetric with respect to the imaginary axis, just as the spectrum of $\mathcal{A}_{a}(\ell)$ in the previous section, but it is no longer symmetric with respect to the real axis, since the operator $\mathcal{A}_{a}(\ell, \gamma)$ is not real. Instead, we have the following result.

Lemma 5.3 Assume that $\gamma \in\left(-\frac{1}{2}, \frac{1}{2}\right]$ and $\gamma \neq 0$. Then the spectrum $\sigma\left(\mathcal{A}_{a}(\ell, \gamma)\right)$ of $\mathcal{A}_{a}(\ell, \gamma)$ is symmetric with respect to the imaginary axis, and $\sigma\left(\mathcal{A}_{a}(\ell, \gamma)\right)=\sigma\left(-\mathcal{A}_{a}(\ell,-\gamma)\right)$.

Proof. The first property is a consequence of the decomposition (5.1) and of the result in [7, Proposition 2.5]. The second property is due to the equality

$$
\mathcal{A}_{a}(\ell, \gamma) \mathcal{S}=-\mathcal{S A}_{a}(\ell,-\gamma)
$$

where $\mathcal{S}$ is the reflection symmetry defined by (4.1).

\subsection{Transverse spectral instability}

We determine now the spectrum of the operator $\mathcal{A}_{a}(\ell, \gamma)$. As a consequence of the second property in Lemma 5.3 we restrict to $\gamma \in\left(0, \frac{1}{2}\right]$. The main result is the following theorem showing the transverse spectral instability of the periodic waves.

Theorem 2 Assume that $\gamma \in\left(0, \frac{1}{2}\right]$ and set $\ell_{c}(\gamma)=\sqrt{3} \gamma(1-\gamma)$. For any a sufficiently small, there exists $\varepsilon_{a}(\gamma)=\gamma^{3 / 2}(1-\gamma)^{3 / 2}|a|\left(1+O\left(a^{2}\right)\right)>0$ such that

(i) for $\left|\ell^{2}-\ell_{c}^{2}(\gamma)\right| \geqslant \varepsilon_{a}(\gamma)$, the spectrum of $\mathcal{A}_{a}(\ell, \gamma)$ is purely imaginary;

(ii) for $\left|\ell^{2}-\ell_{c}^{2}(\gamma)\right|<\varepsilon_{a}(\gamma)$, the spectrum of $\mathcal{A}_{a}(\ell, \gamma)$ is purely imaginary, except for a pair of complex eigenvalues with opposite nonzero real parts.

The remainder of this section is occupied by the proof of this theorem. We rely upon the decomposition (5.1), the result in Corollary 4.5 and the inequality (5.2), and perturbation arguments for linear operators, just as in Section 4 .

Spectrum of $\mathcal{L}_{0}(\ell, \gamma)$ and consequences The linear operator $\mathcal{L}_{a}(\ell, \gamma)$ is a small bounded perturbation of

$$
\mathcal{L}_{0}(\ell, \gamma)=-\left(\partial_{z}+i \gamma\right)^{2}-1-\ell^{2}\left(\partial_{z}+i \gamma\right)^{-2}
$$

Using Fourier series we find

$$
\sigma\left(\mathcal{L}_{0}(\ell, \gamma)\right)=\left\{\mu_{n}(\ell, \gamma)=(n+\gamma)^{2}-1+\frac{\ell^{2}}{(n+\gamma)^{2}} ; n \in \mathbb{Z}\right\}
$$

which shows that 
- the eigenvalues corresponding to the Fourier modes $n \in \mathbb{Z} \backslash\{-1,0\}$ are all positive,

$$
\mu_{n}(\ell, \gamma)>(n+\gamma)^{2}-1 \geqslant \gamma(2+\gamma), \quad \forall n \in \mathbb{Z} \backslash\{-1,0\}
$$

- the eigenvalue

$$
\mu_{-1}(\ell, \gamma)=(1-\gamma)^{2}-1+\frac{\ell^{2}}{(1-\gamma)^{2}}=\gamma^{2}-2 \gamma+\frac{\ell^{2}}{(1-\gamma)^{2}}
$$

corresponding to the Fourier mode $n=-1$ is positive when $\ell^{2}>\ell_{-}^{2}$, where $\ell_{-}^{2}=\gamma(1-\gamma)^{2}(2-\gamma)$, it is zero when $\ell^{2}=\ell_{-}^{2}$, and it is negative when $\ell^{2}<\ell_{-}^{2}$;

- the eigenvalue

$$
\mu_{0}(\ell, \gamma)=\gamma^{2}-1+\frac{\ell^{2}}{\gamma^{2}}
$$

corresponding to the Fourier mode $n=0$ is positive when $\ell^{2}>\ell_{0}^{2}$, where $\ell_{0}^{2}=\gamma^{2}\left(1-\gamma^{2}\right)$, it is zero when $\ell^{2}=\ell_{0}^{2}$, and it is negative when $\ell^{2}<\ell_{0}^{2}$;

(see also Figure $5.2(\mathrm{a})$ ). Here

$$
\ell_{0}^{2}=\gamma^{2}\left(1-\gamma^{2}\right)<\ell_{-}^{2}=\gamma(1-\gamma)^{2}(2-\gamma),
$$

for any $\gamma \in\left(0, \frac{1}{2}\right)$, so that the unperturbed operator has positive spectrum for $\ell^{2}>\ell_{-}^{2}$, one negative

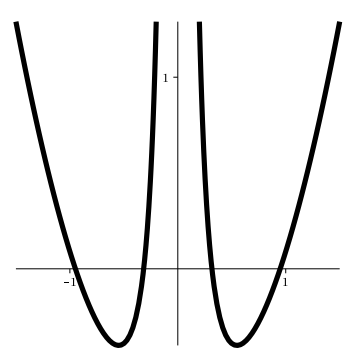

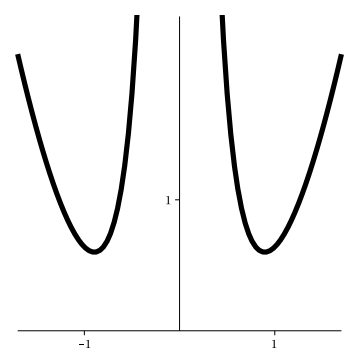

(a)
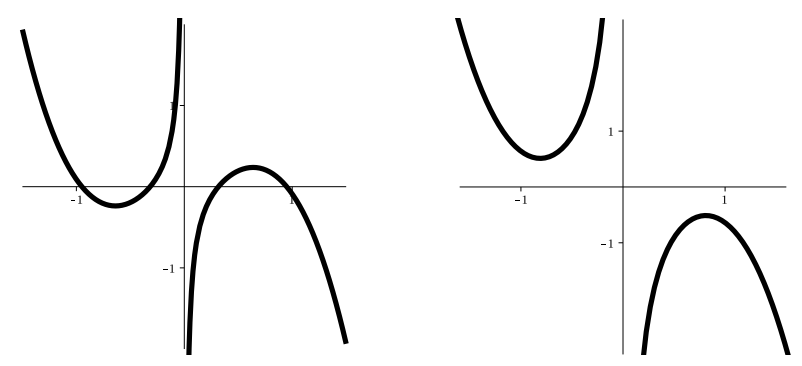

(b)

Figure 5.1: [KP-I equation] (a) Graphs of the map $k \mapsto k^{2}-1+\frac{\ell^{2}}{k^{2}}$ for $\ell=0.3$ and $\ell=0.8$ (from left to right). The eigenvalues of $\mathcal{L}_{0}(\ell, \gamma)$ are found by taking $k=n+\gamma, n \in \mathbb{Z}$. (b) Graphs of the dispersion relation $k \mapsto-k^{3}+k-\frac{\ell^{2}}{k}$ for the same values of $\ell$. The imaginary parts of the eigenvalues of $\mathcal{A}_{0}(\ell, \gamma)$ are found by taking $k=n+\gamma, n \in \mathbb{Z}$. Notice that the zeros of the two maps are the same.

eigenvalue if $\ell_{0}^{2}<\ell^{2}<\ell_{-}^{2}$, and two negative eigenvalues if $0<\ell^{2}<\ell_{0}^{2}$. The following result is an immediate consequence of these properties.

Lemma 5.4 Assume that $\gamma \in\left(0, \frac{1}{2}\right]$. For any $\varepsilon_{*}>0$ there exists $a_{*}>0$, such that the spectrum of $\mathcal{A}_{a}(\ell, \gamma)$ is purely imaginary, for any $\ell$ and a satisfying $\ell^{2} \geqslant \ell_{-}^{2}+\varepsilon_{*}$ and $|a| \leqslant a_{*}$.

Proof. The properties above show that the eigenvalues of the operator $\mathcal{L}_{0}(\ell, \gamma)$ are strictly positive, $\mu_{n}(\ell, \gamma) \geqslant c_{0}(\gamma)$, for some $c_{0}(\gamma)>0$, when $\ell^{2} \geqslant \ell_{-}^{2}+\varepsilon_{*}$. Since $\mathcal{L}_{a}(\ell, \gamma)$ is a small bounded perturbation of $\mathcal{L}_{0}(\ell, \gamma)$ a standard perturbation argument shows that the eigenvalues of $\mathcal{L}_{a}(\ell, \gamma)$ remain strictly positive, provided $a$ is sufficiently small. In particular, $\mathcal{L}_{a}(\ell, \gamma)$ is invertible and $n\left(\mathcal{L}_{a}(\ell, \gamma)=0\right.$, so that $k_{u}\left(\mathcal{A}_{a}(\ell, \gamma)\right)=0$, according to $(5.2)$, which proves the lemma. 
It remains to determine the spectrum of $\mathcal{A}_{a}(\ell, \gamma)$ for $\ell^{2} \in\left(0, \ell_{-}^{2}+\varepsilon_{*}\right)$. We proceed as in Section 4.4, and decompose the spectrum of $\mathcal{A}_{a}(\ell, \gamma)$ into $\sigma_{0}\left(\mathcal{A}_{a}(\ell, \gamma)\right)$ containing a minimal number of eigenvalues, and $\sigma_{1}\left(\mathcal{A}_{a}(\ell, \gamma)\right)$ for which we argue as in Lemma 4.8 to show that it is purely imaginary. More precisely, $\sigma_{1}\left(\mathcal{A}_{a}(\ell, \gamma)\right)$ is such that the restriction of $\mathcal{L}_{a}(\ell, \gamma)$ to the corresponding spectral subspace is positive definite, i.e., it satisfies the inequality (4.3).

Spectrum of $\mathcal{A}_{\mathbf{0}}(\ell, \gamma)$ and decomposition We begin by decomposing the spectrum of the unperturbed operator $\mathcal{A}_{0}(\ell, \gamma)$ in such a way that this decomposition persists for sufficiently small $a$. Using Fourier series again we find

$$
\sigma\left(\mathcal{A}_{0}(\ell, \gamma)\right)=\left\{i \omega_{n}(\ell, \gamma)=-i(n+\gamma)^{3}+i(n+\gamma)-i \frac{\ell^{2}}{n+\gamma} ; n \in \mathbb{Z}\right\}
$$

The location on the imaginary axis of these eigenvalues can be viewed with the help of the dispersion relation

$$
\omega=-k^{3}+k-\frac{\ell^{2}}{k}
$$

(see Figure $5.2(\mathrm{~b})$ ). Notice that the eigenvalues of $\mathcal{A}_{0}(\ell, \gamma)$ which correspond to negative eigenvalues of $\mathcal{L}_{0}(\ell, \gamma)$ are the ones for $n=-1$, if $\ell_{0}^{2}<\ell^{2}<\ell_{-}^{2}$, and for $n=-1$ and $n=0$, if $0<\ell^{2}<\ell_{0}^{2}$. As a consequence of Corollary 4.5 there are these eigenvalues which may lead to instabilities when perturbing the operator $\mathcal{A}_{0}(\ell, \gamma)$. Therefore, the idea of the spectral decomposition is to separate these eigenvalues from the remaining (infinitely many) eigenvalues, which correspond to positive eigenvalues of $\mathcal{L}_{0}(\ell, \gamma)$. Of course this separation is possible as long as there are no collisions with other eigenvalues. Looking for such collisions we find that

- for $\ell_{0}^{2}<\ell^{2}<\ell_{-}^{2}$, the eigenvalue $i \omega_{-1}(\ell, \gamma)$ collides with $i \omega_{0}(\ell, \gamma)$, when

$$
\ell^{2}=\ell_{c}^{2}=3 \gamma^{2}(1-\gamma)^{2}, \quad \ell_{0}^{2}<\ell_{c}^{2}<\ell_{-}^{2}, \quad \forall \gamma \in\left(0, \frac{1}{2}\right),
$$

and it is simple for the other values of $\ell$;

- for $0<\ell^{2} \leqslant \ell_{0}^{2}$, the two eigenvalues $i \omega_{-1}(\ell, \gamma)$ and $i \omega_{0}(\ell, \gamma)$ are simple.

This leads to the following result.

Lemma 5.5 Assume that $\gamma \in\left(0, \frac{1}{2}\right]$. There exist $\varepsilon_{*}>0$ and $c_{*}>0$ such that

(i) for any $\ell$ satisfying $\ell_{c}^{2}+\varepsilon_{*}<\ell^{2}<\ell_{-}^{2}+\varepsilon_{*}$, the spectrum of $\mathcal{A}_{0}(\ell, \gamma)$ decomposes as

$$
\sigma\left(\mathcal{A}_{0}(\ell, \gamma)\right)=\left\{i \omega_{-1}(\ell, \gamma)\right\} \cup \sigma_{1}\left(\mathcal{A}_{0}(\ell, \gamma)\right),
$$

with $\operatorname{dist}\left(i \omega_{-1}(\ell, \gamma), \sigma_{1}\left(\mathcal{A}_{0}(\ell, \gamma)\right)\right) \geqslant c_{*}>0$

(ii) for any $\ell$ satisfying $0<\ell \leqslant \ell_{c}^{2}+\varepsilon_{*}$, the spectrum of $\mathcal{A}_{0}(\ell, \gamma)$ decomposes as

$$
\sigma\left(\mathcal{A}_{0}(\ell, \gamma)\right)=\left\{i \omega_{-1}(\ell, \gamma), i \omega_{0}(\ell, \gamma)\right\} \cup \sigma_{1}\left(\mathcal{A}_{0}(\ell, \gamma)\right)
$$

with $\operatorname{dist}\left(\left\{i \omega_{-1}(\ell, \gamma), i \omega_{0}(\ell, \gamma)\right\}, \sigma_{1}\left(\mathcal{A}_{0}(\ell, \gamma)\right)\right) \geqslant c_{*}>0$.

Perturbation arguments show that this decomposition persists for the operator $\mathcal{A}_{a}(\ell, \gamma)$, for sufficiently small $a$, and we argue as in Section 4.4 to locate the spectrum of $\mathcal{A}_{a}(\ell, \gamma)$. 
Spectrum of $\mathcal{A}_{a}(\ell, \gamma)$ for $\ell_{c}^{2}+\varepsilon_{*}<\ell^{2}<\ell_{-}^{2}+\varepsilon_{*}$ We prove the following result showing that the spectrum of $\mathcal{A}_{a}(\ell, \gamma)$ is purely imaginary for such values of $\ell$, provided $a$ is sufficiently small.

Lemma 5.6 Assume that $\gamma \in\left(0, \frac{1}{2}\right]$. There exist $\varepsilon_{*}>0$ and $a_{*}>0$ such that the spectrum of $\mathcal{A}_{a}(\ell, \gamma)$ is purely imaginary, for any $\ell$ and a satisfying $\ell_{c}^{2}+\varepsilon_{*}<\ell^{2}<\ell_{-}^{2}+\varepsilon_{*}$ and $|a| \leqslant a_{*}$.

Proof. The operator $\mathcal{A}_{a}(\ell, \gamma)$ is a small relatively bounded perturbation of the operator $\mathcal{A}_{0}(\ell, \gamma)$, and there exists $c_{1}>0$ such that

$$
\left\|\mathcal{A}_{a}(\ell, \gamma)-\mathcal{A}_{0}(\ell, \gamma)\right\|_{H^{1} \rightarrow L^{2}} \leqslant c_{1}|a|
$$

for any $\ell>0$ and $a$ sufficiently small. Then by arguing as in the proof of Lemma 4.7, taking into account the spectral decomposition in Lemma 5.5, we conclude that the spectrum of $\mathcal{A}_{a}(\ell, \gamma)$ decomposes as

$$
\sigma\left(\mathcal{A}_{a}(\ell, \gamma)\right)=\sigma_{0}\left(\mathcal{A}_{a}(\ell, \gamma)\right) \cup \sigma_{1}\left(\mathcal{A}_{a}(\ell, \gamma)\right)
$$

where $\sigma_{0}\left(\mathcal{A}_{a}(\ell, \gamma)\right)$ and $\sigma_{1}\left(\mathcal{A}_{a}(\ell, \gamma)\right)$ have the following properties

(i) there exist two open balls $B_{0} \subset B_{1}$ centered at $i \omega_{-1}(\ell, \gamma)$ such that

$$
\sigma_{0}\left(\mathcal{A}_{a}(\ell, \gamma)\right) \subset B_{0}, \quad \sigma_{1}\left(\mathcal{A}_{a}(\ell, \gamma)\right) \subset \mathbb{C} \backslash \overline{B_{1}}
$$

(ii) the spectral projection $\Pi_{a}(\ell, \gamma)$ associated with $\sigma_{0}\left(\mathcal{A}_{a}(\ell, \gamma)\right)$ satisfies $\left\|\Pi_{a}(\ell, \gamma)-\Pi_{0}(\ell, \gamma)\right\|=$ $O(|a|)$;

(iii) the spectral subspace $\mathcal{X}_{a}(\ell, \gamma)=\Pi_{a}(\ell)\left(L_{0}^{2}(0,2 \pi)\right)$ is one dimensional;

(see also Lemma 4.7). This decomposition holds when $\ell_{c}^{2}+\varepsilon_{*}<\ell^{2}<\ell_{-}^{2}+\varepsilon_{*}$ and $|a| \leqslant a_{*}$, for $\varepsilon_{*}$ and $a_{*}$ are sufficiently small.

Next, the eigenvalues in $\sigma_{1}\left(\mathcal{A}_{0}(\ell, \gamma)\right)$ correspond to positive eigenvalues of $\mathcal{L}_{0}(\ell, \gamma)$ ), so that we can argue as in the proof of Lemma 4.8 to conclude that the eigenvalues in $\sigma_{1}\left(\mathcal{A}_{a}(\ell, \gamma)\right)$ are purely imaginary. Finally, the property (iii) shows that $\sigma_{0}\left(\mathcal{A}_{a}(\ell, \gamma)\right)$ consists of one simple eigenvalue. This eigenvalue is the unique eigenvalue of $\mathcal{A}_{a}(\ell, \gamma)$ in the open ball $B_{0}$, and it is purely imaginary since the spectrum of $\mathcal{A}_{a}(\ell, \gamma)$ is symmetric with respect to the imaginary axis. This completes the proof of the lemma.

Spectrum of $\mathcal{A}_{a}(\ell, \gamma)$ for $0<\ell^{2} \leqslant \ell_{c}^{2}+\varepsilon_{*}$ Finally, we prove the following result showing that there is a pair of unstable eigenvalues of $\mathcal{A}_{a}(\ell, \gamma)$, for $\ell$ close to the value $\ell_{c}$ at which the two eigenvalues of $\mathcal{A}_{0}(\ell, \gamma)$ corresponding to the Fourier modes $n=-1$ and $n=0$ collide. This result completes the proof of Theorem 2 .

Lemma 5.7 Assume that $\gamma \in\left(0, \frac{1}{2}\right]$. There exist positive constants $\varepsilon_{*}$, a $a_{*}$, and $\varepsilon_{a}(\gamma)=\gamma^{3 / 2}(1-$ $\gamma)^{3 / 2}|a|\left(1+O\left(a^{2}\right)\right) \leqslant \varepsilon_{*}$ such that the spectrum of $\mathcal{A}_{a}(\ell, \gamma)$ is purely imaginary, if $0<\ell^{2} \leqslant \ell_{c}^{2}+\varepsilon_{*}$ and $\left|\ell^{2}-\ell_{c}^{2}\right| \geqslant \varepsilon_{a}(\gamma)$. If $\left|\ell^{2}-\ell_{c}^{2}\right|<\varepsilon_{a}(\gamma)$ then the spectrum of $\mathcal{A}_{a}(\ell, \gamma)$ is purely imaginary, except for a pair of complex eigenvalues with opposite nonzero real parts. 
Proof. As in the previous case, using the spectral decomposition for $a=0$ in Lemma 5.5, and the arguments in the proof of Lemmas 4.7 and 4.8 we conclude that the spectrum of $\mathcal{A}_{a}(\ell, \gamma)$ decomposes as

$$
\sigma\left(\mathcal{A}_{a}(\ell, \gamma)\right)=\sigma_{0}\left(\mathcal{A}_{a}(\ell, \gamma)\right) \cup \sigma_{1}\left(\mathcal{A}_{a}(\ell, \gamma)\right)
$$

where $\sigma_{1}\left(\mathcal{A}_{a}(\ell, \gamma)\right)$ is purely imaginary, and $\sigma_{0}\left(\mathcal{A}_{a}(\ell, \gamma)\right)$ consists now of two eigenvalues which are the continuation for small $a$ of the eigenvalues $i \omega_{-1}(\ell, \gamma)$ and $i \omega_{0}(\ell, \gamma)$. This decomposition holds for any $\ell$ and $a$ satisfying $0<\ell^{2} \leqslant \ell_{c}^{2}+\varepsilon_{*}$ and $|a| \leqslant a_{*}$, with $\varepsilon_{*}$ and $a_{*}$ sufficiently small. Consequently, it remains to locate the two eigenvalues in $\sigma_{0}\left(\mathcal{A}_{a}(\ell, \gamma)\right)$.

The two eigenvalues $i \omega_{-1}(\ell, \gamma)$ and $i \omega_{0}(\ell, \gamma)$ are simple and the distance between them is strictly positive

$$
\left|i \omega_{-1}(\ell, \gamma)-i \omega_{0}(\ell, \gamma)\right| \geqslant c_{0}
$$

for any $\ell$ outside a neighborhood of $\ell_{c}$, where the eigenvalues collide, and such that $0<\ell^{2} \leqslant \ell_{c}^{2}+\varepsilon_{*}$. A standard perturbation argument then shows that the continuation of this eigenvalues for sufficiently small $a$ is a pair of simple eigenvalues of $\mathcal{A}_{a}(\ell, \gamma)$. Each of these eigenvalues of $\mathcal{A}_{a}(\ell, \gamma)$ is purely imaginary, since the spectrum of $\mathcal{A}_{a}(\ell, \gamma)$ is symmetric with respect to the imaginary axis, just as in the proof of Lemma 5.6. This shows that the spectrum of $\mathcal{A}_{a}(\ell, \gamma)$ is purely imaginary for any $\ell$ outside a neighborhood of $\ell_{c}$.

In order to locate $\sigma_{0}\left(\mathcal{A}_{a}(\ell, \gamma)\right)$ for $\ell$ close to $\ell_{c}$, we proceed as in the proof of Lemma 4.9. We compute successively a basis for the two-dimensional spectral subspace associated with $\sigma_{0}\left(\mathcal{A}_{a}(\ell, \gamma)\right)$, the $2 \times 2$ matrix $M_{a}(\ell, \gamma)$ representing the action of $\mathcal{A}_{a}(\ell, \gamma)$ on this basis, and the eigenvalues of this matrix.

At $a=0$ we choose as basis the two eigenvectors associated with the eigenvalues $i \omega_{0}(\ell, \gamma)$ and $i \omega_{-1}(\ell, \gamma)$,

$$
\xi_{0}^{0}(\ell, \gamma)=1, \quad \xi_{0}^{1}(\ell, \gamma)=e^{-i z}
$$

and then the $2 \times 2$ matrix representing the action of $\mathcal{A}_{0}(\ell, \gamma)$ on this basis is given by

$$
M_{0}(\ell, \gamma)=\left(\begin{array}{cc}
i \omega_{0}(\ell, \gamma) & 0 \\
0 & i \omega_{-1}(\ell, \gamma)
\end{array}\right)
$$

Next, notice that the operator $\mathcal{A}_{a}(\ell, \gamma)$ anti-commutes with the conjugation-reflection symmetry $\mathcal{S}_{c}$ defined by

$$
\mathcal{S}_{c}(u(z))=\overline{u(-z)}
$$

Since

$$
\mathcal{S}_{c} \xi_{0}^{0}(\ell, \gamma)=\xi_{0}^{0}(\ell, \gamma), \quad \mathcal{S}_{c} \xi_{0}^{1}(\ell, \gamma)=\xi_{0}^{1}(\ell, \gamma)
$$

the basis $\left\{\xi_{0}^{0}(\ell, \gamma), \xi_{0}^{1}(\ell, \gamma)\right\}$ can be extended to a basis $\left\{\xi_{a}^{0}(\ell, \gamma), \xi_{a}^{1}(\ell, \gamma)\right\}$, for $a \neq 0$, with the same property. In this basis, the $2 \times 2$ matrix $M_{a}(\ell, \gamma)$ anti-commutes with the conjugation (the map induced by $\left.\mathcal{S}_{c}\right)$, i.e., $M_{a}(\ell, \gamma)=-\overline{M_{a}(\ell, \gamma)}$, which implies that the coefficients of $M_{a}(\ell, \gamma)$ are purely imaginary.

In order to compute the terms of order $a$, we take $\ell=\ell_{c}$, and proceed as in the computation of the vector $\xi_{a}^{1}(0)$ in the proof of Lemma 4.9. Then at order $a$ we find

$$
M_{a}\left(\ell_{c}, \gamma\right)=\left(\begin{array}{cc}
i \omega_{0}\left(\ell_{c}, \gamma\right) & \frac{i}{2} \gamma a \\
\frac{i}{2}(\gamma-1) a & i \omega_{-1}\left(\ell_{c}, \gamma\right)
\end{array}\right)+O\left(a^{2}\right)
$$


Together with the expression of $M_{0}(\ell, \gamma)$, this shows that

$$
M_{a}(\ell, \gamma)=\left(\begin{array}{cc}
i \omega_{0}\left(\ell_{c}, \gamma\right)-i \frac{\varepsilon}{\gamma} & \frac{i}{2} \gamma a \\
\frac{i}{2}(\gamma-1) a & i \omega_{-1}\left(\ell_{c}, \gamma\right)-i \frac{\varepsilon}{\gamma-1}
\end{array}\right)+O(|a|(|\varepsilon|+|a|)),
$$

where $\varepsilon=\ell^{2}-\ell_{c}^{2}$.

Recall that

$$
\ell_{c}^{2}=3 \gamma^{2}(1-\gamma)^{2}, \quad i \omega_{0}\left(\ell_{c}, \gamma\right)=i \omega_{-1}\left(\ell_{c}, \gamma\right)=-2 i \gamma(1-\gamma)(1-2 \gamma)
$$

Then looking for eigenvalues $\lambda$ of the form

$$
\lambda=-2 i \gamma(1-\gamma)(1-2 \gamma)+i X
$$

we find that $X$ is root of the polynomial

$$
P(X)=X^{2}+X\left(\frac{\varepsilon}{\gamma}-\frac{\varepsilon}{1-\gamma}+O\left(a^{2}\right)\right)+\frac{1}{4} \gamma(1-\gamma) a^{2}-\frac{\varepsilon^{2}}{\gamma(1-\gamma)}+O\left(a^{2}\left(|\varepsilon|+a^{2}\right)\right) .
$$

This polynomial has real coefficients, since the matrix $M_{a}(\ell, \gamma)$ is purely imaginary. Here we have also taken in account the fact that these coefficients are even in $a$, due to the equality $P_{a}(z+\pi)=P_{-a}(z)$, which implies that the two roots of the polynomial are the same for $a$ and $-a$. A direct computation shows that the discriminant of this polynomial is

$$
\Delta_{a}(\varepsilon, \gamma)=\frac{\varepsilon^{2}}{\gamma^{2}(1-\gamma)^{2}}-\gamma(1-\gamma) a^{2}+O\left(a^{2}\left(|\varepsilon|+a^{2}\right)\right)
$$

and for any a sufficiently small there exists

$$
\varepsilon_{a}(\gamma)=\gamma^{3 / 2}(1-\gamma)^{3 / 2}|a|\left(1+O\left(a^{2}\right)\right)>0
$$

such that the two roots of the polynomial are real when $|\varepsilon| \geqslant \varepsilon_{a}(\gamma)$, and complex otherwise. This implies that the two eigenvalues of $\mathcal{A}_{a}(\ell, \gamma)$ are purely imaginary when $\left|\ell^{2}-\ell_{c}^{2}\right| \geqslant \varepsilon_{a}(\gamma)$, and complex, with opposite nonzero real parts when $\left|\ell^{2}-\ell_{c}^{2}\right|<\varepsilon_{a}(\gamma)$, which proves the lemma.

\section{KP-II equation: periodic perturbations}

In this section we briefly discuss the KP-II equation, when $\sigma=-1$ in equation (1.1). The functional setup developed in Section 4.1 for periodic perturbations, and in Section 5.1 for non-periodic perturbations remains valid for the KP-II equation. However, it turns out that locating the spectra of the resulting operators is more complicated, because of the qualitatively different behavior of the dispersion relation. We shall restrict here to the case of periodic perturbations, when we have to determine the spectrum of the operator

$$
\mathcal{A}_{a}(\ell)=-\partial_{z} \mathcal{L}_{a}(\ell), \quad \mathcal{L}_{a}(\ell)=-\partial_{z}^{2}-\frac{1}{k_{a}^{2}}\left(\left(1+P_{a}\right) \cdot\right)+\ell^{2} \partial_{z}^{-2},
$$

acting in $L_{0}^{2}(0,2 \pi)$ with domain $H_{\text {per }}^{3}(0,2 \pi) \cap L_{0}^{2}(0,2 \pi)$ (see Corollary 4.2 in Section 4.1 ). We begin by analyzing the spectra of the unperturbed operators $\mathcal{L}_{0}(\ell)$ and $\mathcal{A}_{0}(\ell)$. 
Spectrum of $\mathcal{L}_{0}(\ell) \quad$ Using Fourier series we find that the spectrum of the operator $\mathcal{L}_{0}(\ell)$ acting in $L_{0}^{2}(0,2 \pi)$ is given by

$$
\sigma\left(\mathcal{L}_{0}(\ell)\right)=\left\{\mu_{n}(\ell)=n^{2}-1-\frac{\ell^{2}}{n^{2}} ; n \in \mathbb{Z}^{*}\right\} ;
$$

(see also Figure $6(\mathrm{a})$ ). In contrast to the KP-I equation, the spectrum of $\mathcal{L}_{0}(\ell)$ contains now negative

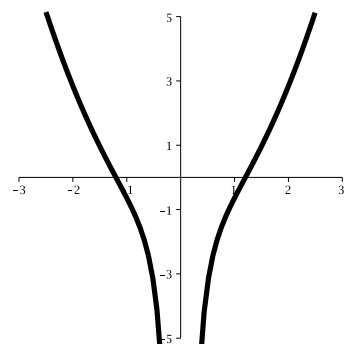

(a)

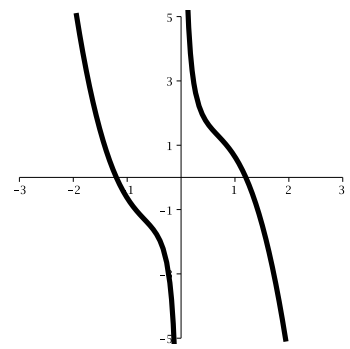

(b)

Figure 6.1: [KP-II equation] (a) Graph of the map $k \mapsto k^{2}-1-\frac{\ell^{2}}{k^{2}}$ for $\ell=0.8$. The eigenvalues of $\mathcal{L}_{0}(\ell)$ are found by taking $k=n, n \in \mathbb{Z}^{*}$. (b) Graph of the dispersion relation $k \mapsto-k^{3}+k+\frac{\ell^{2}}{k}$ for $\ell=0.8$. The imaginary parts of the eigenvalues of $\mathcal{A}_{0}(\ell)$ are found by taking $k=n, n \in \mathbb{Z}^{*}$. Notice that the zeros of the two maps are the same.

eigenvalues, and the number of these eigenvalues increases with $\ell$. Therefore we cannot use the result in Corollary 4.5 to exclude unstable eigenvalues of $\mathcal{A}_{a}(\ell)$ for certain values of $\ell$, e.g., as in Lemma 4.6. However, for a given $\ell$ we can use this corollary to reduce the number of potentially unstable eigenvalues to a finite number, e.g., as in Lemmas 4.8, 5.6, or 5.7, but this number tends to $\infty$ as $\ell \rightarrow \infty$.

Spectrum of $\mathcal{A}_{\mathbf{0}}(\ell)$ The spectrum of the operator $\mathcal{A}_{0}(\ell)$ acting in $L_{0}^{2}(0,2 \pi)$ is given by

$$
\sigma\left(\mathcal{A}_{0}(\ell)\right)=\left\{i \omega_{n}(\ell)=-i n^{3}+i n+i \frac{\ell^{2}}{n} ; n \in \mathbb{Z}^{*}\right\}
$$

(see also Figure $6(\mathrm{~b})$ ). Notice that the dispersion relation

$$
\omega=-k^{3}+k+\frac{\ell^{2}}{k}
$$

is monotonically decreasing on $(-\infty,-1]$ and $[1, \infty)$, so that colliding eigenvalues correspond to Fourier modes with opposite signs. A direct calculation then shows that for any $m, p \in \mathbb{N}^{*}$ the eigenvalues corresponding to the Fourier modes $m$ and $-p$ collide when

$$
\ell^{2}=\ell_{m, p}^{2}=m p\left(m^{2}-m p+p^{2}-1\right) .
$$

Moreover, the corresponding eigenvalues of $\mathcal{L}_{0}(\ell)$ have opposite signs, so that any of these collisions may lead to unstable eigenvalues of the operator $\mathcal{A}_{a}(\ell)$. We can use the arguments presented in the previous sections to reduce the spectral analysis to that of the continuation, for small $a$, of the two colliding eigenvalues, but an additional difficulty comes now from the fact that there are infinitely many values of $\ell$ where two potentially unstable eigenvalues collide. Furthermore, it turns out that in order to determine the location of these two eigenvalues, we need to compute Taylor expansions up to order 
$m+p$ for the resulting $2 \times 2$ matrix. Consequently, the complete spectral analysis seems to be much more difficult in this case. However, we can easily conclude in the case $m=p=1$, which corresponds to values of $\ell$ close to $\ell_{1,1}=0$.

Long wavelength transverse perturbations Restricting to small values of $\ell$, i.e., long wavelength transverse perturbations, we can argue as in Section 4.4 and determine the spectrum of $\mathcal{A}_{a}(\ell)$. In contrast to the KP-I equation, now we show that the spectrum of $\mathcal{A}_{a}(\ell)$ is purely imaginary when $\ell$ and $a$ sufficiently small, i.e., the small periodic waves of the KP-II equation are spectrally stable with respect to long wavelength transverse perturbations.

Lemma 6.1 The spectrum of $\mathcal{A}_{a}(\ell)$ is purely imaginary, for any $\ell$ and a sufficiently small.

Proof. It is enough to replace $\ell^{2}$ by $-\ell^{2}$ in the proofs given in Section 4.4. Then the results in Lemmas 4.7 and 4.8 remain the same, the only change appears in the result of the calculation of the eigenvalues of the $2 \times 2$ matrix $M_{a}(\ell)$ in Lemma 4.9. Upon replacing $\ell^{2}$ by $-\ell^{2}$ we find that the two eigenvalues satisfy

$$
\lambda^{2}=-\ell^{2}\left(\ell^{2}+\frac{1}{12} a^{2}\right)+O\left(a^{2} \ell^{2}\left(\ell^{2}+a^{2}\right)\right)
$$

Consequently, $\lambda^{2}<0$, for $\ell$ and $a$ sufficiently small, which shows that the two eigenvalues are purely imaginary, so that the spectrum of $\mathcal{A}_{a}(\ell)$ is purely imaginary.

\section{Discussion}

We have presented a functional set-up for the study of the transverse spectral stability of the onedimensional periodic waves of the KP equation. Relying upon perturbation arguments for linear operators we have determined the spectra of the operators arising in the spectral problems for the waves of small amplitude of the KP-I equation, and showed that these waves are transversely unstable with respect to perturbations which are either periodic or non-periodic (localized or bounded) in the direction of propagation. For the KP-II equation, the study turns out to be more complicated, due to the 'negative dispersion', which leads to a great number of 'potentially unstable' modes. In this case it is only shown that the periodic waves are transversely spectrally stable with respect to perturbations which are periodic in the direction of propagation and have long wavelengths in the transverse direction. It seems much more difficult to study transverse perturbations in the finite and short wavelength regimes, with this type of arguments, since the location of the potentially unstable eigenvalues is determined by terms of arbitrary high order in the expansion of these eigenvalues for small $a$. In these regimes a numerical computation of the spectrum, using for instance the package described in $[4,5]$, may help to locate the spectrum, and so get more insight in the stability properties of these waves.

This type of approach to transverse spectral stability of periodic waves can be adapted to other two-dimensional dispersive models, e.g., generalized KP equations, Boussinesq systems, equations of Schrödinger type. However, one has to restrict to periodic waves of small amplitude, since we strongly rely upon perturbation arguments for linear operators. For large waves, we mention the approach in [9] which leads to instability criteria, and in the particular case of integrable equations, so also for the KP equation, one could take advantage of the integrability and explicitly compute the spectra of the linear operators (e.g., see [3] for such an approach in the case of the KdV equation). 
Finally, we point out that these results concern only the spectral stability of the periodic waves, the question of nonlinear stability being widely open. Nevertheless, we expect that the recent methods developed in $[11,12]$ for the transverse nonlinear instability of the solitary waves, can be adapted to periodic waves. In particular, these would lead to a transverse nonlinear instability result for the periodic waves of the KP-I equation.

\section{References}

[1] J. C. Alexander, R. L. Pego, and R. L. Sachs. On the transverse instability of solitary waves in the Kadomtsev-Petviashvili equation. Phys. Lett. A 226 (1997), 187-192.

[2] J. Angulo Pava, J. L. Bona, and M. Scialom. Stability of cnoidal waves. Adv. Differential Equations 11 (2006), 1321-1374.

[3] N. Bottman and B. Deconinck. KdV cnoidal waves are spectrally stable. Discrete Contin. Dyn. Syst. 25 (2009), 1163-1180.

[4] B. Deconinck, F. Kiyak, J. D. Carter, and J. N. Kutz. SpectrUW: a laboratory for the numerical exploration of spectra of linear operators. Math. Comput. Simulation 74 (2007), 370-378.

[5] B. Deconinck and J. N. Kutz. Computing spectra of linear operators using the Floquet-Fourier-Hill method. J. Comput. Phys. 219 (2006), 296-321.

[6] M. Haragus. Stability of periodic waves for the generalized BBM equation. Rev. Roumaine Maths. Pures Appl. 53 (2008), 445-463.

[7] M. Haragus and T. Kapitula. On the spectra of periodic waves for infinite-dimensional Hamiltonian systems. Physica D 237 (2008), 2649-2671.

[8] M. Haragus, E. Lombardi, and A. Scheel. Stability of wave trains in the Kawahara equation. J. Math. Fluid Mech. 8 (2006), 482-509.

[9] M. Johnson and K. Zumbrun. Transverse instability of periodic traveling waves in the generalized Kadomtsev-Petviashvili equation. Preprint (2009).

[10] B. B. Kadomtsev and V. I. Petviashvili. On the stability of solitary waves in weakly dispersive media. Soviet Phys. Dokl. 15 (1970), 539-541.

[11] F. Rousset and N. Tzvetkov. Transverse nonlinear instability of solitary waves for some Hamiltonian PDE's. J. Math. Pures Appl. 90 (2008), 550-590.

[12] F. Rousset and N. Tzvetkov. Transverse nonlinear instability for two-dimensional dispersive models. Ann. Inst. H. Poincaré Anal. Non Linéaire 26 (2009), 477-496. 